VOL. $3(1970), 369-373$.

\title{
On the sharpness of a limiting case of the Sobolev imbedding theorem
}

\author{
J. A. Hempel, G. R. Morris and N. S. Trudinger \\ A refinement of the Sobolev imbedding theorem, due to Trudinger, \\ is shown to be optimal in a natural sense.
}

Let $\Omega$ be a bounded domain in Euclidean $n$ space, $E^{n}$. The Sobolev spaces $w_{p}^{k}(\Omega)$, where $k$ is a non-negative integer and $p \geq 1$, consist of those functions in $L_{p}(\Omega)$ whose distributional derivatives of orders up to and including $k$ are also in $L_{p}(\Omega)$ and are Banach spaces under the norm

$$
\|u\|_{k, p}=\sum_{|\alpha| \leq k}\left\|D^{\alpha} u\right\|_{L_{p}(\Omega)} .
$$

The Sobolev imbedding theorem (for the case $k=1$ ) asserts that if $\Omega$ satisfies a cone condition and $p<n$, the space $W_{p}^{l}(\Omega)$ may be continuously imbedded in $L_{q}(\Omega)$ where $q=n p /(n-p)$. If $p>n$, the functions in $\omega_{p}^{I}(\Omega)$ are continuous (after possible redefinition on a set of measure zero). A refinement, proved by Trudinger in [2], shows that the space $W_{n}^{J}(\Omega)$ may be continuously imbedded in the Orlicz space $L_{\phi}(\Omega)$ with defining $N$ function

$$
\phi(t)=e^{|t|^{\frac{n}{n-1}}}-1 .
$$

Received 10 August 1970. 
The purpose of this note is to show that this result is optimal in the sense that the space $L_{\phi}(\Omega)$ above cannot be replaced by any smaller Orlicz space. We let ${ }_{p}^{\circ}(\Omega) \subset W_{p}^{k}(\Omega)$ denote the closure of $c_{0}^{\infty}(\Omega)$ in $W_{p}^{k}(\Omega)$. The reader is referred to [2] for any other relevant definitions and notation.

THEOREM 1. The space ${ }_{n}^{\circ}{ }^{1}(\Omega)$ may not be continuously imbedded in cony Orlicz space $L_{\psi}(\Omega)$ whose defining function $\psi$ increases strictly more rapidly than the function $\phi$ given by (2).

We remark here that $\psi$ increases strictly more rapidly than $\phi$ if for every $\alpha>0, \psi(\alpha \lambda) / \phi(\lambda) \rightarrow \infty$ as $\lambda \rightarrow \infty$. This happens if and only if $L_{\psi}(\Omega) \neq L_{\phi}(\Omega)$. Theorem $I$ is a consequence of the following two lemmas:

LEMMA 1. Let $\phi$ and $\psi$ be $N$ functions, with $\psi$ increasing strictly more rapidly than $\phi$, and suppose that there exists a continuous imbedding of a normed, Zinear space $X$ into $L_{\psi}(\Omega)$. Then $J(u)=\int_{\Omega} \phi(u) d x$ is bounded on bounded subsets of $X$.

Proof. We may identify $X$ and its image $L_{\psi}(\Omega)$ under the imbedding map. Since this mapping is continuous, there is a constant $K \geq 0$ so that $\|u\|_{\psi} \leq K\|u\|_{X}$ for all $u$ in $X$, where $\|u\|_{\psi}$ denotes the Luxemburg norm of $u$, that is

$$
\|u\|_{\psi}=\inf \left\{k>0 ; \int_{\Omega} \psi\left(\frac{u}{k}\right) d x \leq 1\right\} .
$$

Since $\psi$ increases strictly more rapidly than $\phi$, there exists a non-decreasing function of $\lambda \geq 0, N(\lambda)$, satisfying

$$
\phi(t) \leq \psi\left(\frac{t}{K \lambda}\right) \text { for } t \geq N(\lambda) \text {. }
$$

Hence, for any $u$ in $X$ and $\lambda=\|u\|_{X}$, 


$$
\begin{aligned}
\int_{\Omega} \phi(u) d x & \leq \int_{\Omega} \phi(N(\lambda)) d x+\int_{\Omega} \psi\left(\frac{u}{\lambda K}\right) d x \\
& \leq \phi(N(\lambda))|\Omega|+1 .
\end{aligned}
$$

The lemma is thus proved. //

LEMMA 2. Let $A=A(A)$ denote the set of Lipschitz continuous functions on the interval $[0,1]$, vanishing at $x=1$ and satisfying $\int_{0}^{1} x^{n-1}\left|u^{\prime}\right|^{n} d x \leq A$. Then $J(u)=\int_{0}^{1} x^{n-1} e^{|u|^{\frac{n}{n-1}}} d x$ is unbounded on $A$ when $A>n^{n-1}$.

Proof. Let $\rho$ satisfy $0<\rho<1$ and consider a sequence of piecewise linear functions, $u_{k} \in A, k=1,2, \ldots$, satisfying $u_{k}^{\prime}(x)=a_{k j}>0$ for $x \in\left(\rho^{j}, \rho^{j-1}\right), j=1,2, \ldots, k$ and $u_{k}^{\prime}(x)=0$ for $x \in\left(0, \rho^{k}\right)$. Then

$$
\begin{aligned}
\int_{0}^{1} x^{n-1}\left|u_{k}^{\prime}\right|^{n} d x & =\frac{0^{-n}-1}{n} \sum_{j=1}^{k} \rho^{j n} a_{k j}^{n} \\
& =\frac{u^{n}-1}{n} \sum_{j=1}^{k} a_{k j}^{n}
\end{aligned}
$$

where $u=\rho^{-1}$ and $\alpha_{k j}=\rho^{j} a_{k j}$.

$$
\text { Also for } x \leq \rho^{k} \text {, we have }
$$

$$
\begin{aligned}
u_{k}(x)=u_{k}\left(\rho^{k}\right) & =-\left(\rho^{-1}-1\right) \sum_{j=1}^{k} \rho^{j} a_{k j} \\
& =-(u-1) \sum_{j=1}^{k}, \alpha_{k j} .
\end{aligned}
$$

We now choose $\alpha_{k j}$ so that $\sum \alpha_{k j}$ is maximised subject to the constraint $\left(u^{n}-1\right) \sum \alpha_{k_{j}}^{n}=A n$; that is, we choose $\alpha_{k 1}=\alpha_{k 2}=\ldots=\alpha_{k k}=\left(\frac{A n}{u^{n}-1}\right)^{\frac{1}{n}}-\frac{1}{n}$, so that 
(8)

$$
u_{k}\left(\rho^{k}\right)=-(u-1)\left(\frac{A n}{u^{n}-1}\right)^{\frac{1}{n}} \frac{n-1}{n} .
$$

Therefore, by (7) and (8),

$$
\begin{aligned}
J\left(u_{k}\right) & \geq \int_{0}^{\rho^{k}} x^{n-1} e^{|u|^{\frac{n}{n-1}}} d x \\
& =\frac{1}{n} e^{\beta k}
\end{aligned}
$$

where $B=B(U)=(U-1)^{\frac{n}{n-1}}\left(\frac{A n}{U^{n}-1}\right)^{1 /(n-1)}-n \log u$.

$$
\text { Since } \beta(U) /(U-1) \text { approaches } A^{\frac{1}{n-1}}-n>0 \text { as } U \rightarrow 1 \text {, it is }
$$

possible to choose $U>I$ to guarantee $\beta>0$. It then follows that $J\left(u_{k}\right)$ is unbounded. //

We remark here that if in the statement of Lemma 2, we assume $A<n^{n-1}$, then $J(u)$ is bounded on A. For then we have

$$
\begin{aligned}
|u(x)| & \leq \int_{x}^{1}\left|u^{\prime}(t)\right| d t \\
& \leq\left(\int_{x}^{1} \frac{d t}{t}\right)^{1-\frac{1}{n}}\left(\int_{0}^{1} t^{n-1}\left|u^{\prime}(t)\right|^{n} d t\right)^{\frac{1}{n}} \text { by Hölder's inequality, } \\
& \leq A^{\frac{1}{n}}\left(\log \frac{1}{x}\right)^{1-\frac{1}{n}}
\end{aligned}
$$

and consequentiy

$$
J(u) \leq \int_{0}^{1} x^{n-1-A^{n-1}} d x=\left(n-A^{\frac{1}{n-1}}\right)^{-1} .
$$

To complete the proof of Theorem 1, we may without loss of generality take $\Omega$ as the unit sphere in $E^{n}$ and consider spherically symmetric 
functions, $u=u(r)$, only. Then

$$
\int_{\Omega}|D u|^{n} d x=w_{n} \int_{0}^{1} x^{n-1}\left|u_{r}\right|^{n} d r, \int_{\Omega} e^{|u|^{\frac{n}{n-1}}} d x=w_{n} \int_{0}^{1} r^{n-1} e^{|u|^{\frac{n}{n-1}}} d r,
$$

and Theorem $I$ consequently follows from Lemmas 1 and 2 . As a consequence of Lemma 2, we also have

THEOREM 2. The space ${ }_{n}^{\circ}{ }_{n}^{1}(\Omega)$ may not be compactly imbedded in the Orlicz space $L_{\phi}(\Omega)$ where $\phi$ is given by (2).

Note that Theorem 1 is also a consequence of Theorem 2. In view of Theorem 2 and Theorem 3 of [2], it would be of interest to study the unique solvability in ${ }_{2}^{0}{ }_{2}^{1}(\Omega)$ of differential equations such as

$$
\Delta u+u e^{u^{2}}=0
$$

in two dimensions. Hempel [1] has made some progress in this direction.

\section{References}

[1] J.A. Hempel, "Superlinear variational boundary value problems and non-uniqueness", Doctoral thesis, University of New England, 1970.

[2] N.S. Trudinger, "On imbedding into Orlicz spaces and some applications", J. Math. Mech. 17 (1967), 473-484.

\footnotetext{
University of New England, Armidale, New South Wales;

University of New England, Armidale, New South Wales;

University of Queensland, St Lucia, Queensland.
} 